\title{
ANTIFIBRILLATORY ACTIONS OF BEPRIDIL AND BUTYL-MDI, TWO INTRACELLULAR CALCIUM ANTAGONISTS
}

\author{
JOSEPH J. LYNCH ${ }^{1}$, RALF G. RAHWAN ${ }^{2}$ and BENEDICT R. LUCCHESI ${ }^{1 *}$ \\ ${ }^{\prime}$ Department of Pharmacology, University of Michigan Medical School, Ann Arbor, Michigan; and ${ }^{2}$ College of Pharmacy, Ohio State \\ University, Columbus, Ohio, U.S.A.
}

Received 8 January 1985, accepted 22 January 1985

J.J. LYNCH, R.G. RAHWAN and B.R. LUCCHESI, Antifibrillatory actions of bepridil and butyl-MDI, two intracellular calcium antagonists, European J. Pharmacol. 111 (1985) 9-15.

The antifibrillatory and electrophysiologic actions of bepridil and butyl-methylenedioxyindene (BU-MDI), two intracellular calcium antagonists, were examined in anesthetized dogs. The administration of bepridil $(1.0-10.0 \mathrm{mg} / \mathrm{kg}$ i.v.) significantly increased the electrical threshold for ventricular fibrillation determined during unobstructed coronary flow, and was associated with a significant decrease in ventricular excitability and a progressive depression in ventricular myocardial conduction. BU-MDI $(3.0-30.0 \mathrm{mg} / \mathrm{kg}$ i.v.) did not significantly alter ventricular fibrillation thresholds during unobstructed coronary flow, nor did it significantly alter electrophysiologic properties such as ventricular excitability, conduction or refractoriness. The administration of either bepridil $(10 \mathrm{mg} / \mathrm{kg}$ i.v.) or BU-MDI $(30 \mathrm{mg} / \mathrm{kg}$ i.v.), however, resulted in significant increases in the ventricular fibrillation thresholds determined during transient myocardial ischemia, restoring the threshold values to corresponding non-ischemic levels. These results suggest that an inhibition of the action and/or availability of intracellular calcium may play a role in the antifibrillatory actions of BU-MDI and bepridil during transient ischemia.

Methylenedioxyindene (MDI) Antiarrhythmics Calcium antagonist Ventricular fibrillation Bepridil Myocardial ischemia

\section{Introduction}

Bepridil (CERM-1978) (B-(2-methylpropoxy)methyl-N-phenyl-N-phenylmethyl)-1-pyrrolidineethanamine hydrochloride) and BU-MDI (2-nbutyl 3-dimethylamino-5,6-methylenedioxyindene hydrochloride) are structurally distinct compounds which have been characterized as intracellular calcium antagonists'. An inhibition of the action and/or availability of intracellular calcium has been proposed to contribute toward the smooth muscle relaxant and cardiodepressant actions of

\footnotetext{
* To whom all correspondence should be addressed: Department of Pharmacology, M6322 Medical Science Building I, The University of Michigan Medical School, Ann Arbor, Michigan 48109, U.S.A.
}

both bepridil (Vogel et al., 1979; Mras and Sperelakis, 1981) and BU-MDI (Rahwan et al., 1977; Lynch and Rahwan, 1982; Heaslip and Rahwan, 1983). Pronounced cellular accumulations and slow, delayed (presumably intracellular) components of efflux have been demonstrated for bepridil (Mras and Sperelakis, 1982; Pang and Sperelakis, 1983) and BU-MDI (Lynch et al., 1983) in isolated cardiac and/or vascular smooth muscle preparations. Mitochondria and sarcoplasmic reticulum have been identified as potential sites of action for both bepridil (Younes et al., 1977, 1981; Younes and Constantin, 1980) and BU-MDI (Matlib et al., 1981; Lynch et al., 1983).

In a variety of experimental animal models, BU-MDI (Piascik et al., 1979; Lynch et al., 1981, 1982) and bepridil (Kane and Winslow, 1980; Marshall and Muir, 1981; Jackson et al., 1985; 
Torres et al., 1984) display a wide spectrum of antiarrhythmic activity. The purpose of the present investigation was to examine the antifibrillatory properties of these two compounds by determining their effects upon the ventricular fibrillation threshold in the anesthetized dog during both unobstructed coronary flow and during transient ischemia. The results of this investigation suggest that an inhibition of the action and/or availability of calcium might play a role in the antifibrillatory actions of these two 'intracellular calcium antagonists' during transient regional myocardial ischemia.

\section{Materials and methods}

\subsection{Surgical preparation}

Male mongrel dogs (12.0-15.0 kg) were anesthetized with intravenous sodium pentobarbital, $30 \mathrm{mg} / \mathrm{kg}$. A cuffed endotracheal tube was inserted and the animals were ventilated with room air using a Harvard respirator. A cannula was inserted into the left common carotid artery to allow continuous monitoring of arterial pressure and a cannula was inserted into the left external jugular vein to facilitate drug administration.

A left thoracotomy was performed in the fourth intercostal space and the heart was suspended in a pericardial cradle. The left anterior descending coronary artery was dissected free from the surrounding myocardium distal to the second diagonal branch, and a loose ligature passed beneath the artery. An epicardial bipolar electrode $(1 \mathrm{~mm}$ diameter silver electrodes embedded $3 \mathrm{~mm}$ apart in acrylic) was sutured to the right ventricular surface adjacent to the site of coronary artery isolation. This electrode was used for the delivery of trains of current pulses for the ventricular fibrillation threshold determinations. A second epicardial bipolar electrode was sutured to the left ventricular surface in the distribution of the left circumflex coronary artery for the recording of local ventricular activation. A bipolar plunge electrode (25 gauge stainless steel, $5 \mathrm{~mm}$ in length, 2 $\mathrm{mm}$ apart) was sutured into the left ventricular myocardium near the tip of the left atrial appen- dage for determination of excitation thresholds and refractory periods. Heart rate was controlled with atrial pacing $(4 \mathrm{~ms}$ duration pulses at twice diastolic threshold) via a bipolar clip electrode placed on the left atrial appendage.

\subsection{Measurement of electrophysiologic parameters} and determination of ventricular fibrillation thresholds

Electrocardiographic intervals and electrophysiologic parameters were determined immediately before ventricular fibrillation threshold determinations. Electrocardiographic intervals were measured during sinus rhythm. A paced QT interval was determined during atrial pacing $(3 \mathrm{~Hz})$. Ventricular excitation thresholds, effective refractory periods and activation times were determined during atrial pacing $(3.5 \mathrm{~Hz})$. The ventricular excitation threshold was defined as the minimum current required to produce a conducted ventricular beat at a stimulus duration of $4 \mathrm{~ms}$ delivered 250 $\mathrm{ms}$ after the R-wave of the lead II electrocardiogram. The ventricular effective refractory period was the longest $\mathbf{R}$ to stimulus interval at which a $2 \times$ threshold stimulus ( $4 \mathrm{~ms}$ duration) failed to produce a conducted ventricular beat. Ventricular activation time, the interval between the Q-wave of the lead II electrocardiogram and the largest deflection of the local ventricular electrogram (QEG), was measured on a Tektronix model 5111 oscilloscope.

Ventricular fibrillation thresholds were determined during atrial pacing $(3.5 \mathrm{~Hz})$. Trains of 4 ms duration square-wave pulses $(60 \mathrm{~Hz}, 200 \mathrm{~ms}$ duration) were delivered to the right ventricle beginning $50 \mathrm{~ms}$ after the peak of the R-wave. The output of a Grass S-88 stimulator, Grass SIU-5 stimulus isolation unit and a Grass constant current unit was triggered from the R-wave of the lead II electrocardiogram using a Tektronix 565 oscilloscope and a type 3A8 operational amplifier. Current intensity was increased in $1.0 \mathrm{~mA}$ increments to $10 \mathrm{~mA}$, then in $2.0 \mathrm{~mA}$ increments to 20 $\mathrm{mA}$, and in $5 \mathrm{~mA}$ increments until ventricular fibrillation occurred. Three trials were performed at each current intensity with 8- to 10-beat intervals between each trial. The lowest current inten- 
sity producing ventricular fibrillation was defined as the ventricular fibrillation threshold. When fibrillation occurred, the heart was defibrillated immediately using a capacitor-discharge direct current defibrillator charged to 10 joules.

\subsection{Experimental protocol and drug administration}

Before drug or vehicle administration, two control determinations of electrophysiologic parameters and ventricular fibrillation thresholds were made 15 min apart during unobstructed coronary flow. Thereafter, one control (pretreatment) determination of ventricular fibrillation threshold during a temporary occlusion of the left anterior descending coronary artery was made. This latter determination was started $2 \mathrm{~min}$ after occlusion of the artery and was completed by the 6th min of occlusion. After control pretreatment determinations had been made, three groups of six dogs each received bepridil $(1.0,3.0$ and $10.0 \mathrm{mg} / \mathrm{kg}$ i.v., cumulatively), BU-MDI $(3.0,10.0$ and $30.0 \mathrm{mg} / \mathrm{kg}$ i.v., cumulatively) or matching volumes of vehicle for the aforementioned drug dosages $(2.0,6.0$ and $20.0 \mathrm{ml}$ of $10 \% \mathrm{v} / \mathrm{v}$ ethanol in distilled water, i.v., cumulatively). Each indicated treatment dosage or volume was achieved by a $10 \mathrm{~min}$ infusion and was followed $15 \mathrm{~min}$ later by redetermination of the electrophysiologic parameters and the ventricular fibrillation threshold during unobstructed coronary flow. After the three groups of dogs had received a cumulative $20 \mathrm{ml}$ of vehicle, $30 \mathrm{mg} / \mathrm{kg}$ BU-MDI or $10 \mathrm{mg} / \mathrm{kg}$ bepridil, final determinations of the electrophysiologic parameters and the ventricular fibrillation threshold during unobstructed coronary flow were made. Fifteen minutes thereafter, a second (post-treatment) determination of ventricular fibrillation threshold during acute coronary occlusion was made. Determination of ventricular fibrillation threshold during transient regional ischemia was performed only twice in the protocol for each preparation (one determination pretreatment; the second post-treatment determination after full drug or vehicle administration) in order to avoid the potentially deleterious effects of repeated coronary artery occlusions and reperfusions upon the electrophysiologic state of the preparation. Dosage ranges for be- pridil and BU-MDI in this study were based upon the activities of these compounds in previously reported antiarrhythmic evaluations (see Introduction for references).

\subsection{Statistical analysis}

Data are expressed as means \pm S.E.M. For all parameters excluding fibrillation thresholds during coronary occlusion, the percentage change from the mean control response was computed for each dog. Following arcsine transformations, a one-way analysis of variance followed by a Dunnett's test for multiple comparisons to one control (vehicle) group was used to detect significant differences between either drug-treated group and the volume-matched vehicle-treated group. Within each treatment group, a paired Student's t-test was used to compare pre- and post-treatment values of ventricular fibrillation threshold during coronary artery occlusion. $\mathrm{P}<0.05$ was the criterion of significance in all cases.

\section{Results}

\subsection{Effect of vehicle administration on ventricular fibrillation threshold and electrophysiologic parame- ters}

The cumulative administration of up to $20 \mathrm{ml}$ of vehicle $(10 \% \mathrm{v} / \mathrm{v}$ ethanol in distilled water) did not significantly alter ventricular fibrillation thresholds determined during unobstructed left anterior descending coronary flow. The ventricular fibrillation threshold values for cumulative volumes of vehicle are compared graphically to corresponding values for the bepridil-treated group in fig. 1 . A slight but consistent decrease in the ventricular fibrillation threshold determined during tansient coronary occlusion was observed after cumulative vehicle treatment (fig. 2), indicating the development of an increased vulnerability toward ventricular fibrillation during transient ischemia over the course of the experiment. The pretreatment values for sinus heart rate, mean arterial pressure and electrophysiologic parameters for the vehicle treatment group did not differ significantly from the 


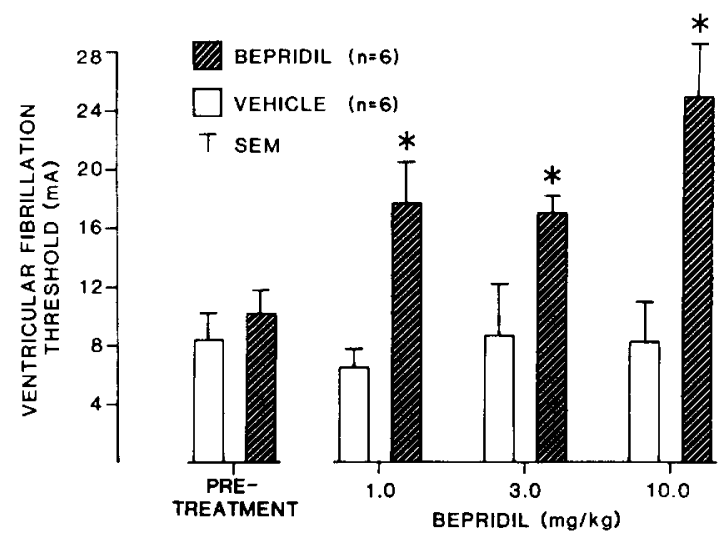

Fig. 1. Effects of bepridil (striped bars) or corresponding volumes of vehicle (open bars) on the ventricular fibrillation threshold during unobstructed coronary flow. Depicted values are means \pm S.E.M. with $n=6$. $\left(^{*}\right)$ Indicates a significant $(P<0.05)$ alteration from pretreatment value.

pretreatment values observed for the bepridil (table 1) and BU-MDI treatment groups, nor did vehicle administration significantly alter these parameters.

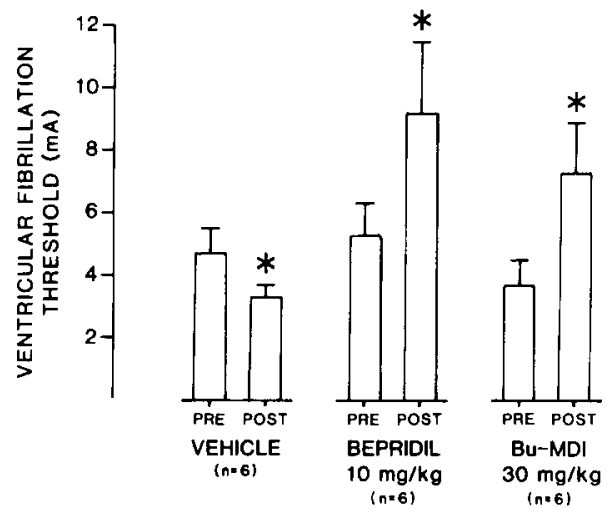

Fig. 2. Values for ventricular fibrillation threshold determined during transient regional ischemia before (pre) and after (post) the administration of vehicle (left panel), bepridil (center panel) or BU-MDI (right panel). The depicted values are means \pm S.E.M. with $n=6$. $\left({ }^{*}\right)$ Indicates a significant $(P<0.05)$ post vs. pre difference.

\subsection{Effect of bepridil administration on ventricular fibrillation threshold and electrophysiologic parame- ters}

The cumulative administration of bepridil, 1.0 to $10.0 \mathrm{mg} / \mathrm{kg}$ i.v., resulted in a significant elevation in ventricular fibrillation thresholds de-

\section{TABLE 1}

The effects of bepridil administration on electrophysiologic and hemodynamic parameters in pentobarbital-anesthetized, open-chest dogs. All values represent means \pm S.E.M. with $n=6$.

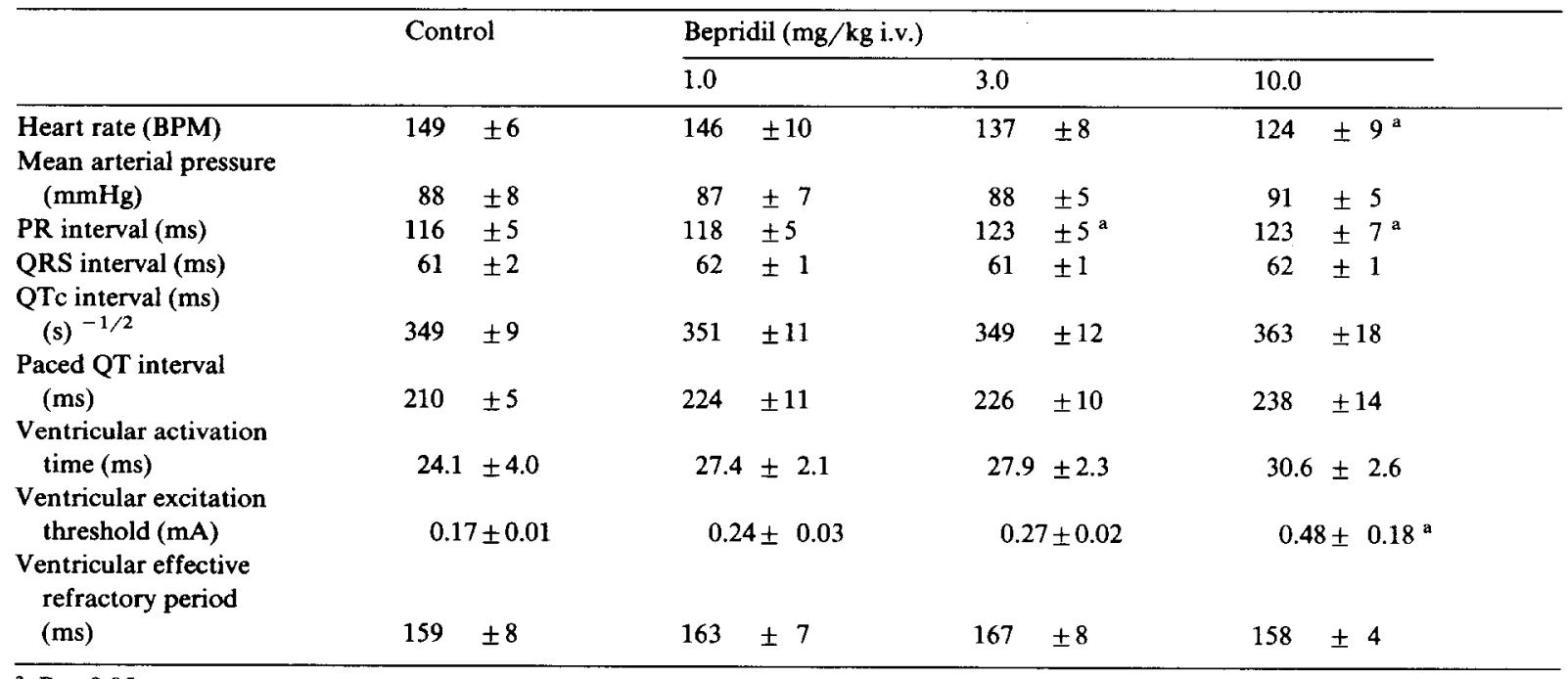

\footnotetext{
${ }^{\mathrm{a}} \mathrm{P}<0.05$.
} 
termined during unobstructed coronary flow (fig. 1). Dosages of 1.0 and $3.0 \mathrm{mg} / \mathrm{kg}$ bepridil produced near equivalent $80-90 \%$ increases in the fibrillation threshold, while the cumulative 10 $\mathrm{mg} / \mathrm{kg}$ dose produced a $170 \%$ increase in fibrillation threshold. The administration of $10 \mathrm{mg} / \mathrm{kg}$ bepridil also was associated with a significant elevation in the ventricular fibrillation threshold determined during transient regional myocardial ischemia (fig. 2).

The electrophysiologic effects of bepridil administration are summarized in table 1. The cumulative administration of bepridil was associated with increases in the ventricular excitation threshold (attaining statistical significance at 10 $\mathrm{mg} / \mathrm{kg}$ ) as well as trends toward increasing ventricular activation times, QTc and paced QT intervals. A slight increase in ventricular refractoriness was noted at $3 \mathrm{mg} / \mathrm{kg}$ bepridil. The administration of bepridil was associated with a slight but consistent increase in the PR interval (significant at 3.0 and $10.0 \mathrm{mg} / \mathrm{kg}$ ) and a reduction in the sinus heart rate (significant at $10 \mathrm{mg} / \mathrm{kg}$ ).

\subsection{Effect of $B U-M D I$ on ventricular fibrillation threshold and electrophysiologic parameters}

The cumulative administration of BU-MDI, 3.0 to $30.0 \mathrm{mg} / \mathrm{kg}$ i.v., resulted in only slight and insignificant alterations in the ventricular fibrillation threshold determined during unobstructed coronary flow. The cumulative administration of $30.0 \mathrm{mg} / \mathrm{kg} \mathrm{BU}-\mathrm{MDI}$, however, resulted in a consistent and significant elevation in the ventricular fibrillation threshold determined during transient regional myocardial ischemia (fig. 2). BU-MDI administration did not significantly alter sinus heart rate, electrocardiographic intervals or electrophysiologic parameters determined during unobstructed coronary flow.

\section{Discussion}

4.1. Ventricular fibrillation threshold determinations during unobstructed coronary flow - effects of bepridil and $B U-M D I$ administration

In the present evaluation, the administration of bepridil $(1.0-10.0 \mathrm{mg} / \mathrm{kg}$ i.v.) significantly increased the electrical threshold for ventricular fibrillation determined during unobstructed coronary flow. A concomitant decrease in ventricular myocardial excitability (increased excitation thresholds) and a progressive depression in ventricular myocardial conduction (increased ventricular activation times) accompanied the administration of bepridil. These latter electrophysiologic effects are suggestive of the actions of class I antiarrhythmic agents, inhibitors of fast channel sodium entry during myocardial cell depolarization (Zipes, 1984). The antifibrillatory actions of bepridil in this setting may be due, at least in part, to these electrophysiological effects. In vitro bepridil has been demonstrated to reduce the intensity of the fast inward sodium current in sheep Purkinje fibers $\left(5 \times 10^{-6}\right.$ to $2 \times 10^{-5} \mathrm{M}$ bepridil) and in guinea pig ventricular muscle $\left(2-8 \times 10^{-5}\right.$ $M$ bepridil), suggesting that the compound displays class I electrophysiologic effects at the cellular level (Kane and Winslow, 1980).

The administration of BU-MDI $(3.0-30.0 \mathrm{mg} / \mathrm{kg}$ i.v.) did not significantly alter the ventricular fibrillation threshold determined during unobstructed coronary flow. In this dosage range, BUMDI produced only a slight and insignificant increase in the ventricular excitation threshold and was associated with a slight progressive decrease in ventricular activation times (i.e. a slight enhancement in myocardial conduction).

\subsection{Ventricular fibrillation threshold determinations during transient ischemia - effects of bepridil and $B U-M D I$ administration}

It is well established that transient myocardial ischemia increases the degree of dispersion in recovery of ventricular myocardium, and that this increased electrophysiologic inhomogeneity is reflected in a decrease in ventricular fibrillation 
threshold (Han, 1969; Axelrod et al., 1975). Accordingly, in the present study, the control pretreatment values for the ventricular fibrillation threshold determined during a transient occlusion of the left anterior descending coronary artery were consistently and significantly less than corresponding pretreatment threshold values determined during unobstructed coronary flow, indicating an increased electrophysiologic instability during ischemia. A consistent and significant decrease in the value for ventricular fibrillation threshold determined during transient ischemia was observed after cumulative vehicle administration. This increased vulnerability toward ventricular fibrillation during ischemia observed in the control group may well reflect metabolic alterations in the preparations during the course of the protocol (Moore and Spear, 1975). Despite this enhanced vulnerability toward ventricular fibrillation during transient ischemia, the administration of either bepridil $(10 \mathrm{mg} / \mathrm{kg}$ i.v.) or BU-MDI $(30 \mathrm{mg} / \mathrm{kg}$ i.v.) resulted in a significant increase in ventricular fibrillation threshold (compared to the appropriate pre-drug value) during coronary occlusion. For both bepridil and BU-MDI, post-treatment ventricular fibrillation thresholds determined during transient ischemia did not differ significantly from corresponding predrug values determined during unobstructed coronary flow.

The ability of BU-MDI to significantly elevate the ventricular fibrillation threshold in the ischemic state at a dose that significantly altered neither fibrillation thresholds in the non-ischemic state nor electrophysiologic properties such as ventricular excitability, conduction or refractoriness raises the possibility that the ability of BU-MDI to alter the availability and/or action of cytosolic calcium may underlie its antifibrillatory activity during transient ischemia. Likewise, the ability of bepridil to modulate cytosolic calcium might also contribute toward the elevation in the ventricular fibrillation threshold during transient ischemia in the presence of the drug. It certainly may not be precluded that other pharmacologic actions of these compounds, most particularly the actions of bepridil upon myocardial conduction and excitability, might underlie, in part, the antifibrillatory actions of these compounds during transient ischemia. Taken together, however, the foregoing results suggest the possibility that an inhibition of the action and/or availability of intracellular calcium may play a role in the antifibrillatory actions of BU-MDI and of bepridil during transient myocardial ischemia. Such a protective, antifibrillatory action of these intracellular calcium antagonists' during ischemia is fully consistent with the concept that intracellular calcium accumulation plays a primary role in the pathophysiology of myocardial ischemia (Trump et al., 1982; Nayler, 1983).

\section{Acknowledgements}

This work was supported by the National Institutes of Health, Heart, Lung and Blood Institute, Grant \# HL-05806-24 and by a grant from the McNeil Pharmaceutical Company. Joseph J. Lynch is a Postdoctoral Fellow of the American Heart Association of Michigan.

\section{References}

Axelrod, P.J., R.L. Verrier and B. Lown, 1975, Vulnerability to ventricular fibrillation during acute coronary arterial occlusion and release, Am. J. Cardiol. 36, 776.

Han, J., 1969, Ventricular vulnerability during acute coronary occlusion, Am. J. cardiol. 242, 857.

Heaslip, R.J. and R.G. Rahwan, 1983, Norepinephrine-induced contractions of the rat aorta in the absence of extracellular calcium - II. Effects of calcium antagonists, Gen. Pharmacol. 14, 505.

Jackson, C.V., S.E. Mitsos, P.J. Simpson, E.M. Driscoll and B.R. Lucchesi, 1985, Effects of bepridil on regional and global myocardial ischemia/reperfusion-induced injury, Pharmacology (in press).

Kane, K.A. and E. Winslow, 1980, Antidysrhythmic and electrophysiological effects of a new antianginal agent, bepridil, J. Cardiovasc. Pharmacol. 2, 193.

Lynch, J.J. and R.G. Rahwan, 1982, Absence of blocking effects on cardiac slow calcium channels by the intracellular calcium antagonist 2-n-propyl-3-dimethylamino-5,6-methylenedioxyindene, Can. J. Physiol. Pharmacol. 60, 841.

Lynch, J.J., R.G. Rahwan, R.J. Brumbaugh and D.D. Witiak, 1982, Effects of tertiary and quaternary derivatives of aminomethylenedioxyindenes on some experimental arrhythmias, Can. J. Physiol. Pharmacol. 60, 1636.

Lynch, J.J., R.G. Rahwan and D.T. Witiak, 1981, Effects of 2-substituted 3-dimethylamino-5,6-methylenedioxyindenes on calcium-induced arrhythmias, J. Cardiovasc. Pharmacol. 3, 49 . 
Lynch, J.J., R.G. Rahwan, D.T. Witiak and F.D. Cazer, 1983, Intracellular localization of the calcium antagonist propylmethylenedioxyindene in cardiac tissue, Gen. Pharmacol. 14, 571.

Marshall, R.J. and A.W. Muir, 1981, The beneficial actions of bepridil in acute myocardial infarction in anesthetized dogs, Br. J. Pharmacol. 73, 471.

Matlib, M.A., P.L. Vaghy, J.D. Johnson, D. Rebman, R.G. Rahwan and A. Schwartz, 1981, Protective effect of calcium channel blocking drugs and of calmodulin inhibitor trifluoperazine on inorganic phosphate-induced swelling and inhibition of oxidative phosphorylation of heart mitochondria, 8th Int. Congr. Pharmacol., Tokyo, Abst. 1025.

Moore, E.N. and J.F. Spear, 1975, Ventricular fibrillation threshold - its physiological and pharmacological importance, Arch. Intern. Med. 135, 446.

Mras, S. and N. Sperelakis, 1981, Bepridil (CERM-1978) and verapamil depression of contractions of rabbit aortic rings, Blood Vessels 18, 196.

Mras, S. and N. Sperelakis, 1982, Comparison of $\left[{ }^{3} \mathrm{H}\right]$ bepridil and $\left[{ }^{3} \mathrm{H}\right]$ verapamil uptake into rabbit aortic rings, $J$. Cardiovasc. Pharmacol. 4, 777.

Nayler, W.G., 1983, Calcium and cell death, European Heart J. 4, C33.

Pang, D.C. and N. Sperelakis, 1983, Nifedipine, diltiazem, bepridil and verapamil uptakes into cardiac and smooth muscles, European J. Pharmacol. 87, 199.

Piascik, M.F., M.T. Piascik, D.T. Witiak and R.G. Rahwan, 1979, Pharmacologic evaluation of new calcium antagonists: 2-substituted 3-dimethylamino-5,6-methylenedioxyindenes.
Antiarrhythmic effects, Can. J. Physiol. Pharmacol. 57, 1350.

Rahwan, R.G., M.M. Faust and D.T. Witiak, 1977, Pharmacological evaluation of new calcium antagonists: 2-substituted 3-dimethylamino-5,6-methylenedioxyindenes, J. Pharmacol. Exp. Ther. 201, 126.

Torres, V., J. Felder, R. Hoch, D. Tepper, D. Weiner and J. Somberg, 1984, The antiarrhythmic action of bepridil in normokalemic and hypokalemic dogs, Fed. Proc. 43, 842.

Trump, B.F., I.K. Berezesky and R.A. Cowley, 1982, The cellular and subcellular characteristics of acute and chronic injury with emphasis on the role of calcium, in: Pathophysiology in Shock, Anoxia and Ischemia, eds. R. Cowley and B. Trump (Williams and Wilkins, Baltimore) p. 6.

Vogel, S., R. Crampton and N. Sperelakis, 1979, Blockade of myocardial slow channels by bepridil (CERM-1978), J. Pharmacol. Exp. Ther. 210, 378.

Younes, A. and M. Constantin, 1980, Effects of bepridil on mitochondrial ATPase reactions, Biochem. Pharmacol. 29, 1135.

Younes, A., C. Fontanarava and J.-M. Schneider, 1981, Effects of bepridil on the Ca-dependent ATPase activity of sarcoplasmic reticulum, Biochem. Pharmacol. 30, 2979.

Younes, A., N. Moins and M. Habert, 1977, Bepridil, a new effector of oxidative phosphorylations, Biochemie 59, 73.

Zipes, D.P., 1984, Management of cardiac arrhythmias: Pharmacological electrical and surgical techniques, in: Heart Disease, ed. E. Braunwald (W.B. Saunders Co., Philadelphia) p. 648. 